\title{
Digital Total Variation filtering as postprocessing for Chebyshev Pseudospectral Methods for Conservation Laws
}

\author{
Scott A. Sarra \\ Marshall University
}

August 15, 2005

\begin{abstract}
Digital total variation filtering is analyzed as a fast, robust, postprocessing method for accelerating the convergence of pseudospectral approximations that have been contaminated by Gibbs oscillations. The method, which originated in image processing, can be combined with spectral filters to quickly post-process large data sets with sharp resolution of discontinuities and with exponential accuracy away from the discontinuities.
\end{abstract}

keywords: Gibbs phenomenon, Numerical Partial Differential Equations, Pseudospectral methods, Digital Total Variation filtering, conservation laws.

\section{Introduction}

Pseudospectral methods are based on assuming that an unknown PDE solution $u$ can be represented by a global, interpolating partial sum,

$$
u_{N}(x)=\sum_{k=0}^{N} a_{k} \phi_{k}(x) .
$$

Taking $\phi$ to be the Chebyshev polynomials $\phi_{k}(x)=T_{k}(x)=\cos (k \arccos x)$, as we do in this work, gives a Chebyshev approximation method or in the context of numerical PDEs, a Chebyshev pseudospectral (CPS) method. If $\phi$ are taken to be the Legendre polynomials or trigonometric polynomials (Fourier pseudospectral method), the basic ideas of the post-processing are 
essentially the same. Detailed information on pseudospectral methods may be found in the standard references $[1,4,14,33]$.

It is well-documented that for sufficiently smooth solutions, the CPS method is spectrally accurate. However, in the case of the numerical solution of nonlinear hyperbolic conservation laws, discontinuities (edges) in the form of shocks and rarefaction waves may develop independently of the smoothness of the initial conditions and destroy the exponential accuracy of the method. Away from the discontinuities, the convergence is slowed to a crawl. Near the discontinuities there are $\mathcal{O}(1)$ oscillations which do not decrease with $N$. This is known as the Gibbs phenomenon. Additionally, the application of the CPS method to a nonlinear conservation law results in an unstable method. It is necessary to add spectral viscosity to the method in order to obtain a stable approximation which converges to the exact entropy solution [29]. This can be done in several different ways, with each way being labelled a particular type of spectral viscosity [30] method. In our numerical examples, we have used the super spectral viscosity (SSV) method of [24], which for a conservation law in one space dimension, can be stated as

$$
\frac{\partial}{\partial t} u_{N}+\frac{\partial}{\partial x} f\left(u_{N}\right)=\varepsilon(-1)^{s+1} Q^{2 s} u_{N}
$$

where $Q=\sqrt{1-x^{2}} \frac{\partial}{\partial x}$ is a viscosity operator. Dimensional splitting can be used to handle higher dimension conservation laws. The SSV method can be viewed as applying a weak filter to the CPS method. It can be applied via the spectral filtering framework that is described later in this section, although it does not satisfy the definition of a spectral filter. The result is a stable method, but the weak filtering does not remove the Gibbs oscillations from the SSV approximation.

Over the last 20 years much research has focused on applying spectral methods to PDE problems with discontinuous solutions. The goal of such research efforts has been either the recovery of the spectral accuracy that is achieved in the smooth case or the acceleration of the slowed convergence. Methods have been developed to recover spectral accuracy in smooth regions up to within a distance of each edge. The reconstruction methods may or may not require the location of edges. Methods for locating edges in spectral data in one and two dimensions are described in $[9,10]$. Three types of post-processing methods are spectral filtering, spectral mollification, and Gegenbauer reconstruction.

The simplest approach for lessening the effects of the Gibbs phenomena is to use a spectral filter of order $p$. The spectral partial sum (1) is filtered 


$$
F_{N} u_{N}(x)=\sum_{k=0}^{N} \sigma\left(\frac{k}{N}\right) a_{k} T_{k}(x)
$$

where $\sigma(\eta)$ is an order $p$ spectral filter as defined in [34]. Without knowledge of edge locations, a spectral filter of sufficiently high order $p$ restores spectral accuracy at points sufficiently far away from a discontinuity. However edges are not resolved sharply and smearing is present is the neighborhood of discontinuities. Many different filter functions are available, but perhaps the most versatile and widely used filter is the exponential filter

$$
\sigma(\eta)=\exp \left(-\alpha|\eta|^{p}\right), \quad p \text { even. }
$$

For (1) to numerically satisfy the definition of a spectral filter, the parameter $\alpha$ is taken to be $\alpha=-\ln \varepsilon$ where $\varepsilon$ is machine epsilon.

Another class of method for the removal of the Gibbs phenomena which originated in [20] is the spectral mollification method. The method is also referred to as a local reconstruction procedure. Spectral mollification involves applying a two parameter family of filter to the physical space interpolant (1). The method can recover spectral accuracy up to within a neighborhood of each discontinuity. As described in [20], the method does not incorporate the location of edges. The method results in a reconstructed function that is free of Gibbs oscillations but with smearing at the discontinuities. By incorporating the edge locations into the algorithm, optimal and in some cases adaptive parameter choices can be made to allow the spectral mollification method to be more accurate near discontinuities [8,31]. Recently in [32], optimal spectral filters and mollifiers have been developed by abandoning the restriction that the filter/mollifier have compact support in frequency and physical space and instead require that the filters/mollifiers have optimal joint frequency-physical localization.

The most promising method to date for the removal of the Gibbs phenomena is the Gegenbauer Reconstruction Procedure (GRP) [19, 15, 16, 17, 18]. The GRP is capable of recovering spectral accuracy up to the locations of the discontinuities. The GRP must know the exact location of the edges. Despite a solid theoretical underpinning, the GRP has proven difficult to implement in practice. The GRP is plagued by round-off errors and a high computational cost. The GRP is difficult to implement in higher dimensions largely due to the fact that each piecewise smooth subregion must be located and then two un-optimized parameters must be specified in each subregion. Some progress has been made towards specifying optimal values 
of the GRP parameters [21], although the optimal values may not be applicable due to round-off errors. Recently, promising reprojection methods that use a more stable Gibbs alternative basis then the Gegenbauer basis have been described [13]. An Inverse Gegenbauer Reconstruction Procedure (IGRP) has recently been described [28] that can accurately reconstruct a discontinuous function from its pseudo-spectral coefficients. However, it was shown in [26] that the IGRP is unable to reconstruct an oscillatory function, such as a CPS numerical PDE approximation, that is polluted with Gibbs oscillations. The IGRP requires the pseudospectral coefficients of a piecewise continuous, but non-oscillatory, function for the reconstruction to be accurate.

In dimensions greater than one, it has proven difficult to robustly implement post-processing techniques that require the exact location of the edges. If the function to be reconstructed has features not aligned with the cartesian grid, resolution will be lost as edge detection methods will only locate the neighborhoods of the discontinuities in this situation [12]. If one or more user defined parameters must be specified in each smooth subregion, the method is further complicated. Ideally, a post-processing method should be capable of removing Gibbs oscillations while sharply resolving edges and should do so without knowledge of the locations of the edges. Additionally it should not require that excessive free parameters be specified. The method should accomplish this in a computationally efficient manner amenable for extension to higher dimensions. A method meeting these criteria could then be implemented as a "black box" post-processing method. Such methods have been developed in the field of image processing and can be adapted to post-process the numerical solution of conservation laws by pseudospectral methods.

\section{Digital TV filtering}

Variational based PDE restoration methods, such as total variation (TV) filtering [23], have become one of the most important tools in image processing. The methods assume that the images are defined on a continuous domain and a continuous variational functional is constructed from which an Euler-Lagrange equation is derived. The resulting differential equations are then discretized by existing numerical PDE methods on a cartesian grid. Another approach is to digitize the entire methodology. This approach starts directly with a discrete variational problem and works with data on a general discrete domain, a graph. The digitized approach is more flexible as 
irregularly shaped domains and scattered data points can be handled with ease. One such digitized method is the digital total variation (DTV) filter which was developed in [5] and further analyzed in [25]. In the context of numerical PDEs, it has previously been applied to steady solutions of conservation laws computed by second-order Lax-Wendroff methods in [2] and [3]. Next, the pertinent facts about the DTV filter from are summarized and the reader is referred to $[5,25]$ for details.

Let $[\Omega, G]$ be a finite set $\Omega$ of nodes and a dictionary of edges $G$. General vertices are denoted by $\alpha, \beta, \cdots$. The notation $\alpha \sim \beta$ indicates that $\alpha$ and $\beta$ are linked by an edge. All the neighbors of $\alpha$ are denoted by

$$
N_{\alpha}=\{\beta \in \Omega \mid \beta \sim \alpha\} .
$$

Assume a noisy data distribution $u^{0}$ on $\Omega$ that is an approximation to a clean data distribution $u$ that is contaminated by noise which in this case is the Gibbs oscillations. The noise is removed by solving an unconstrained minimization problem that minimizes a fitted TV energy,

$$
E_{\lambda}^{T V}(u)=\sum_{\alpha \in \Omega}\left|\nabla_{\alpha} u\right|_{a}+\frac{\lambda}{2}\left\|u-u^{0}\right\|_{\Omega}^{2} .
$$

The solution of the minimization problem is a system of nonlinear equations which can be shown to have a unique solution that depends on a parameter $\lambda$ and on $u^{0}$. The nonlinear system can be solved by a linearized Jacobi iteration as

$$
u_{\alpha}^{[n+1]}=\sum_{\beta \sim \alpha} h_{\alpha \beta} u_{\beta}^{[n]}+h_{\alpha \alpha} u_{\alpha}^{0} .
$$

The filter coefficients are defined as

$$
h_{\alpha \beta}(u)=\frac{w_{\alpha \beta}(u)}{\lambda+\sum_{\gamma \sim \alpha} w_{\alpha \gamma}(u)}
$$

and

$$
h_{\alpha \alpha}(u)=\frac{\lambda}{\lambda+\sum_{\gamma \sim \alpha} w_{\alpha \gamma}(u)}
$$

where

$$
w_{\alpha \beta}(u)=\frac{1}{\left|\nabla_{\alpha} u\right|_{a}}+\frac{1}{\left|\nabla_{\beta} u\right|_{a}} .
$$

The regularized location variation or strength at any node $\alpha$ is defined as

$$
\left|\nabla_{\alpha} u\right|_{a}=\left[\sum_{\beta \in N_{\alpha}}\left(u_{\beta}-u_{\alpha}\right)^{2}+a^{2}\right]^{1 / 2} .
$$


The parameter $\lambda$ is a fitting parameter corresponding to a Lagrange multiplier in the variational problem that has been digitized. The regularization parameter $a$ is a small constant to avoid a zero local variation and to ensure the stability of the algorithm. We have used $a=10^{-8}$ in our numerical examples, but we note that choosing $a$ such that $10^{-4} \leq a \leq 10^{-16}$ was also effective. Using a regularized local variation as opposed to a local variation, i.e. (11) with $a=0$, does not alter the important properties of the method such as the existence and uniqueness of a solution and the edge detecting ability of the algorithm [25]. The iteration can be initialized by setting $u^{[0]}=u^{0}$. Typically, after the first several iterations, the corrections to the previous iterations are very small. Stopping the iteration after a fixed number of iterations, e.g. 50 is usually sufficient. Otherwise, an effective stopping criteria is for the relative $L^{1}$ residual between two consecutive iterations to be less than some tolerance, i.e.,

$$
\frac{\left\|u^{[k+1]}-u^{[k]}\right\|_{L^{1}}}{\left\|u^{[k]}\right\|_{L^{1}}} \leq t o l
$$

In the examples, $t o l=10^{-8}$ produced good results.

An estimation of the optimal fitting parameter for a current signal $u^{[n]}$ is $[5]$

$$
\lambda^{[n]} \approx \frac{1}{\sigma^{2}} \frac{1}{N+1} \sum_{\alpha \in \Omega} \sum_{\beta \sim \alpha} w_{\alpha \beta}\left(u_{\beta}^{[n]}-u_{\alpha}^{[n]}\right)\left(u_{\alpha}^{[n]}-u_{\alpha}^{0}\right)
$$

where $\sigma^{2}$ is the variance of the noise. The filtering process can be started with

$$
\lambda=\frac{1}{\sigma^{2}},
$$

and then updated every several steps with 13 . However, if the initial $\lambda$ is used throughout the entire filtering process the results are typically just as good as if $\lambda$ had been updated every several steps. A wide range of $\lambda$ results in an accurate post-processing.

The digital TV filter can be seen to be a lowpass filter as

$$
h_{\alpha \alpha}+\sum_{\beta \in N_{\alpha}} h_{\alpha \beta}=1, \quad \alpha \in \Omega .
$$

Because of the lowpass filter property the digital TV filter satisfies the maximum principle

$$
\min _{\beta} u_{\beta}^{0} \leq u_{\alpha}^{[n]} \leq \max _{\beta} u_{\beta}^{0}
$$


at each node $\alpha$. Unlike other post-processing algorithms, the DTV filter has built-in edge detection. For a properly chosen regularization constant $\lambda$, e.g. as specified by equation (14), a jump in the data will be indicated by the weights $w_{\alpha \beta}$ being small compared to $\lambda$. This in turn causes $h_{\alpha \alpha}$ to be near one which leads to a large portion of the original data being retained as the filter is applied. On the other hand, in smooth regions, the weights are large compared to $\lambda, h_{\alpha \beta}$ will be large and $h_{\alpha \alpha} \ll 1$ which leads to the oscillations in the data being smoothed.

\section{DTV filtering as pseudospectral post-processing}

Below, six options for applying the DTV filter as pseudospectral postprocessing are described. The results are illustrated by interpolating the step function

$$
f(x)=\left\{\begin{array}{lr}
1 & -1 \leq x \leq 0, \\
0 & 0<x \leq 1
\end{array}\right.
$$

known at $N+1$ points $x_{i}=-\cos \left(\frac{\pi i}{N}\right), i=0,1, \ldots, N$ to 200 evenly spaced points on $[-1,1]$ using (1). In the left image figure 1 the expected slow convergence of the Chebyshev partial sum to the piecewise continuous function is illustrated. Unless otherwise specified we have used 50 iterations to reach the post-processed function. The execution times of the algorithm were measured on a $3.00 \mathrm{GHz}$ pentium 4 with Windows XP using Matlab 6.5. The six options are:

1. Digital TV filter, constant $\lambda$. The most straightforward way to apply the DTV filter is by specifying $\lambda$ as (14). In the right image of figure 1 we see that the acceleration of the convergence in the neighborhood of the discontinuity is impressive compared to the Chebyshev approximation in the left image of figure 1 and that the discontinuity is very sharply resolved. However, the convergence away from the discontinuity is not as impressive. The relatively small oscillations away from the discontinuity are more difficult for the DTV filter to remove than the large oscillations near the jump since we have used $\lambda=15$ which corresponds to the large oscillations in the vicinity of the jump.

If more than 50 iterations are taken very small corrections to the postprocessed approximation continue to be made for a large number of iterations. If the iteration continues until a steady state is reached, the smaller oscillations near the boundary are more effectively removed. Faster converging iterative algorithms such as Gauss-Siedel could be 
used to reduce the number of iterations required for a steady state to be reached.

2. Digital TV filter, adaptive optimal lambda. The DTV filter is applied using $\lambda=1 / \sigma^{2}$ for the first iteration and then updating $\lambda$ in additional iterations by (13). In our numerical experiments, this did not improve the results of option 1.

3. Local lambda. In the right image of figure 1 a global constant $\lambda=$ 15 was used which corresponds to the strong oscillations around the discontinuity. This value allowed the jump to be sharply resolved and from the error plot we can see that a local spectral accuracy has been recovered in the region surrounding the discontinuity. However, near the boundaries where only mild oscillations exist, a larger value of $\lambda$ is required to remove the small oscillations in the given number of iterations. This suggests the use of a variable of $\lambda$. For each point $\alpha_{i}$ of the vector to be post-processed, $\lambda_{i}$ is estimated based on the strength of the oscillations in an interval of radius $M$ points, centered when possible and one-sided when necessary. Using a locally defined $\lambda$ did not produce a more accurate post-processed approximation than option 1 . The failure of specifying $\lambda$ locally has been documented in [2] in the application to a Lax-Wendroff solution to a conservation law. The same authors report some success with a locally specified $\lambda$ in [3] if edge detection is used. However, in our results we have found this not to be the case as described in the next option.

4. Incorporating edge detection. The DTV filter is implemented in smooth regions found by external edge detection methods and $\lambda$ is specified in each smooth subregion. The DTV filter automatically recognizes and accounts for edges. Regions of homogeneous noise strength are more important than regions of smoothness. In our numerical experiments, incorporating edge detection did not improve the algorithm, and in most cases resulted in discontinuities not being as sharply resolved as when the DTV filter was applied globally. The large oscillations near a discontinuity were not resolved accurately unless the filter had knowledge of the large oscillations on the other side of the discontinuity.

5. Digital TV and weak spectral filter. Motivated by the success of the DTV filter for post-processing the CPS SSV solutions of conservation laws which follow in the numerical examples section, a weak 
spectral filter is first applied and then the DTV filter. The CPS SSV method can be interpreted as applying a very weak filter (although it does not satisfy the definition of a spectral filter) to the CPS approximation. As a result, the CPS SSV approximation is largely free of the very small oscillations that the DTV filter does not remove very well from the step function approximation. In figure the left image of 2 the improved convergence away from the discontinuity is evident with only a very slight smearing of edge.

6. Hybrid TV filter/spectral filter. The hybrid method uses the DTV filter where the maximum absolute difference of function values in a neighborhood is larger than $t_{l} l_{p p}$ and a spectral filter otherwise. In figure the right image of figure 2 the hybrid method is shown to reach machine accuracy away from the discontinuity and to resolve the edge sharply where the DTV filter is applied near the discontinuity. The hybrid method resulted in the most accurate postprocessing of approximations to which spectral viscosity had not been applied, i.e. interpolation problems and linear hyperbolic PDE solutions.

The discontinuity in the step function is in the center of the domain. However, the DTV filter is just as effective with discontinuities located closer to boundaries. The filter only requires that the location of the discontinuity be at least one grid point from the boundary.

\subsection{Two-dimensional data}

For two-dimensional pseudospectral grids, there exists more than one way to define $N_{\alpha}$. One is to consider at a node $\alpha_{i, j}$ four neighboring points, $N_{\alpha}^{4}=\left\{\alpha_{i, j+1}, \alpha_{i+1, j}, \alpha_{i, j-1}, \alpha_{i-1, j}\right\}$. Another is an eight point neighborhood, $N_{\alpha}^{8}=\left\{\alpha_{i, j+1}, \alpha_{i+1, j+1}, \alpha_{i+1, j}, \alpha_{i+1, j-1}, \alpha_{i, j-1}, \alpha_{i-1, j-1}, \alpha_{i-1, j}, \alpha_{i-1, j+1}\right\}$. No advantage has been found when using the larger neighborhood and all numerical results use the four point neighborhood $N_{\alpha}^{4}$.

Consider the function

$$
f(x, y)= \begin{cases}3 & x, y<0 \text { and }|x|+|y| \geq 1 \\ 2 & x \geq 0 \text { and } y<0 \text { or } \\ & x<0 \text { and } y \geq 0 \text { or } \\ & x<0 \text { and } y<0 \text { and }|x|+|y|<1 \\ 1 & x, y>0 \text { and } x^{2}+y^{2}<0.5 \\ 0 & \text { otherwise. }\end{cases}
$$




\begin{tabular}{|c|c|c|c|c|c|}
\hline$N$ & $(0.3,0.3)$ & $(-0.57,-0.57)$ & $(-0.45,-0.45)$ & $(0.75,0.75)$ & $(-0.50,0.50)$ \\
\hline 40 & $1.4 \mathrm{e}-2$ & $5.2 \mathrm{e}-2$ & $3.5 \mathrm{e}-2$ & $2.8 \mathrm{e}-2$ & $1.4 \mathrm{e}-2$ \\
80 & $4.5 \mathrm{e}-2$ & $4.8 \mathrm{e}-2$ & $4.3 \mathrm{e}-2$ & $1.8 \mathrm{e}-2$ & $9.4 \mathrm{e}-3$ \\
160 & $4.9 \mathrm{e}-2$ & $9.5 \mathrm{e}-3$ & $3.5 \mathrm{e}-2$ & $6.2 \mathrm{e}-3$ & $4.2 \mathrm{e}-3$ \\
\hline
\end{tabular}

Table 1: Chebyshev interpolation errors

\begin{tabular}{|c|c|c|c|c|c|}
\hline$N$ & $(0.3,0.3)$ & $(-0.57,-0.57)$ & $(-0.45,-0.45)$ & $(0.75,0.75)$ & $(-0.50,0.50)$ \\
\hline 40 & $2.7 \mathrm{e}-3$ & $2.9 \mathrm{e}-2$ & $1.2 \mathrm{e}-2$ & $2.7 \mathrm{e}-3$ & $6.7 \mathrm{e}-3$ \\
80 & $4.2 \mathrm{e}-5$ & $1.2 \mathrm{e}-5$ & $1.1 \mathrm{e}-3$ & $1.0 \mathrm{e}-3$ & $7.8 \mathrm{e}-6$ \\
160 & $1.1 \mathrm{e}-5$ & $8.3 \mathrm{e}-6$ & $3.0 \mathrm{e}-7$ & $2.7 \mathrm{e}-5$ & $3.6 \mathrm{e}-6$ \\
\hline
\end{tabular}

Table 2: DTV filter post-processed CPS errors, $\lambda=9,50$ iterations.

The function contains features not aligned with the cartesian grid. Such a function presents potential difficulties for post-processing methods that need the location of the edges pinpointed.

Table 1 records at five points the convergence of a Chebyshev approximation used to interpolate function (18) to a $200 \times 200$ uniform grid from a $N \times N$ tensor product grid constructed from the one-dimensional grid given by $x_{i}=-\cos \left(\frac{\pi i}{N-1}\right), i=0,1, \ldots, N-1$. The points include locations near discontinuities and in smooth regions. A contour plot of the CPS approximation for $N=160$ is in the left image of figure 3 . Table 2 lists the errors of the TV post-processed approximation using a global $\lambda=9$ and 50 iterations. The post-processing at the 40,000 nodes took 4 seconds. The convergence has been accelerated both near and away from the discontinuities. The convergence can further be accelerated if the CPS approximation is first filtered with a weak $p=26$ order spectral filter and then filtered with the DTV filter. The error information is in table 3. The hybrid DTV/spectral filter

\begin{tabular}{|c|c|c|c|c|c|}
\hline$N$ & $(0.3,0.3)$ & $(-0.57,-0.57)$ & $(-0.45,-0.45)$ & $(0.75,0.75)$ & $(-0.50,0.50)$ \\
\hline 40 & $6.9 \mathrm{e}-2$ & $1.2 \mathrm{e}-2$ & $5.8 \mathrm{e}-2$ & $4.6 \mathrm{e}-4$ & $8.9 \mathrm{e}-3$ \\
80 & $1.6 \mathrm{e}-3$ & $2.4 \mathrm{e}-4$ & $1.2 \mathrm{e}-3$ & $7.4 \mathrm{e}-5$ & $3.9 \mathrm{e}-5$ \\
160 & $1.2 \mathrm{e}-4$ & $4.5 \mathrm{e}-7$ & $7.5 \mathrm{e}-8$ & $1.7 \mathrm{e}-6$ & $4.8 \mathrm{e}-8$ \\
\hline
\end{tabular}

Table 3: Spectral/DTV filter post-processed CPS errors, $\lambda=9$, 50 iterations, and exponential filter $p=26$. 


\begin{tabular}{|c|c|c|c|c|c|}
\hline$N$ & $(0.3,0.3)$ & $(-0.57,-0.57)$ & $(-0.45,-0.45)$ & $(0.75,0.75)$ & $(-0.50,0.50)$ \\
\hline 40 & $2.7 \mathrm{e}-3$ & $2.9 \mathrm{e}-2$ & $1.2 \mathrm{e}-2$ & $2.7 \mathrm{e}-3$ & $6.7 \mathrm{e}-3$ \\
80 & $4.2 \mathrm{e}-5$ & $1.2 \mathrm{e}-5$ & $1.1 \mathrm{e}-3$ & $5.5 \mathrm{e}-7$ & $1.2 \mathrm{e}-6$ \\
160 & $1.1 \mathrm{e}-5$ & $8.3 \mathrm{e}-6$ & $3.0 \mathrm{e}-7$ & $4.0 \mathrm{e}-14$ & $1.2 \mathrm{e}-13$ \\
\hline
\end{tabular}

Table 4: Hybrid, $\lambda=9$ and 50 iterations, $p=4$, tol $_{p p}=10 \mathrm{e}-3$

gives an exponentially accurate post-processing at the two points away from the the discontinuity while accelerating the convergence at points near the discontinuities and sharply resolves the edges. The hybrid results are in table 4 and in the right image of figure 3 .

\section{Numerical Results}

In this section the DTV filter is used to post-process the CPS SVV approximations to nonlinear conservation laws on the nonuniform pseudospectral grid [22],

$$
x_{i}=\frac{\arcsin [-\gamma \cos (i \pi / N)]}{\arcsin \gamma}, \quad i=0,1, \ldots, N \text { and } 0<\gamma<1 .
$$

In two-dimensions a tensor product grid is used. The small oscillations that were present in the interpolation problems are largely absent for the CPS SSV approximations as they have been removed by the mild filtering provided by the application of the SSV. The DTV filter can be applied to the CPS SSV approximations without need for additional spectral filtering.

\subsection{1d conservation law}

The one-dimensional fluidized bed equations are

$$
u_{t}+f(u)_{x}=s(u)
$$

where

$$
u=\left(\begin{array}{c}
\alpha \\
m
\end{array}\right), \quad f=\left(\begin{array}{c}
m \\
m^{2} / \alpha+F(\alpha)
\end{array}\right), \quad s=\left(\begin{array}{c}
0 \\
b(\alpha, m)
\end{array}\right) .
$$

The variable $\alpha(x, t)$ denotes the concentration of particles by volume, the particle momentum is represented by $m(x, t)=\alpha v$, and $v(x, t)$ is the particle velocity. A detailed description of the model can be found in references [6] 
and [7]. If the source term is neglected, an exact solution to the Riemann problem for the homogeneous system can be found and is described in [7]. A detailed description of the Chebyshev SSV solution of the fluidized bed equations may be found in [27].

First we consider the system with the source term set to zero and initial conditions of $v(x, 0)=0$ for all $x$ in a domain of $[-0.2,0.2]$ and $\alpha(x, 0)=0.3$ if $x<0$ and $\alpha(x, 0)=0.55$ if $x \geq 0$. The solution consists of a left-moving shock wave and a right moving rarefaction wave. The left image of figure 4 shows the $N=127$ Chebyshev SSV approximation of $\alpha(x, 0.5)$ versus the exact solution on a grid specified by equation (19) with $\gamma=0.99$. The right image of figure 4 shows the digital TV post-processed (solid dots) $\alpha(x, 0.5)$ vs. the exact solution (solid). The post-processing parameters were $\lambda=105$ and $t o l=10^{-8}$ which leads to 524 iterations taking less than one second. Both the sharp front at the shock and the rarefaction wave are well-resolved.

Next consider system (20) with the source term. An exact solution to the problem with the source term is not known. The initial condition is the state of uniform fluidization, i.e., the state when the solids phase is suspended by gas phase by a minimum gas flow. The solutions of the system feature "slugs", or pockets of gas, that move up vertically (along the $x$-axis) through the particle phase of the bed. It is known that the formation of the slugs corresponds mathematically to the formation of a shock. In the plot of the concentration, the slugging process is visible as rapid variations in the concentration as the slugs travel up through the bed. At the sudden changes in concentration, the Gibbs oscillations are present in the CPS SSV solution and in one area cause un-physical negative concentration approximations. Any post-processing method used on the solutions of the fluidized bed model must be able to both sharply resolve shocks and preserve the fine scale structure of the narrow slugs. In [27] the CPS SSV solutions are postprocessed by the Gegenbauer reconstruction procedure. Post-processing the solutions via the GRP method proved difficult as first a large number of shocks of differing magnitude, but in close proximity, had to be located by edge detection methods. Then the two parameters of the GRP had to be selected in each smooth subinterval until a "good" post-processing was achieved. On the other hand, the DTV filter does not require edge detection, as it is built-in, and only one globally applied parameter need be specified.

Figure 5 shows the $N=512$ Chebyshev SSV approximation of the concentration at time $t=2.95$ on a grid specified by equation (19) with $\gamma=0.995$. Also in figure 5 the digital TV post-processed (solid dots) concentration solution is shown. The post-processing parameters was $\lambda=35$ with 40 iterations taking less than one second. After post-processing, the 
physically unrealistic concentrations $\alpha<0$ have all been replaced by $\alpha \geq 0$ and the shocks have been well resolved with oscillations being removed at the top and at the bottom of the slugs without excessive smearing of the small scale feature slugs.

\subsection{Two-dimensional conservation law}

The two-dimensional shallow water equations are

$$
u_{t}+f(u)_{x}+g(u)_{y}=0
$$

where

$$
u=\left(\begin{array}{c}
h \\
v_{1} h \\
v_{2} h
\end{array}\right), \quad f=\left(\begin{array}{c}
v_{1} h \\
v_{1}^{2} h+\frac{1}{2} g h^{2} \\
v_{1} v_{2} h
\end{array}\right), \quad g=\left(\begin{array}{c}
v_{2} h \\
v_{1} v_{2} h \\
v_{2}^{2} h+\frac{1}{2} g h^{2}
\end{array}\right) .
$$

The variable $h=h(x, y, t)$ represents the height of the free surface, $v_{1}(x, y, t)$ is the fluid velocity in the $x$-direction, $v_{2}(x, y, t)$ is the fluid velocity in the $y$-direction, and $g$ is the acceleration due to gravity.

The test problem is the dam break problem for a circular dam of radius $R=0.44$ which uses the initial conditions $v_{1}(x, y, 0)=0, v_{2}(x, y, 0)=0$, and

$$
h(x, y, 0)= \begin{cases}10 & x^{2}+y^{2} \leq R^{2} \\ 3 & x^{2}+y^{2}>R^{2}\end{cases}
$$

An analytical solution is not known.

The Chebyshev SSV solution on a grid specified by equation (19) with $\gamma=0.99$ is advanced to time $t=0.03$. DTV filtering is applied with $\lambda=5$ and 40 iterations. Since the solution should be radially symmetric, we look at a cross section of the CPS SSV solution and post-processed solution for $x=0$. In figure 6 a cross section of the oscillatory SSV solution of the height is shown in solid and the DTV post-processed solution is shown as solid dots. The edges are sharply resolved. The results can be compared with a Legendre SV approximation and post-processing by a spectral mollification method using edge detection in [11].

\section{Conclusions}

DTV filters were designed to remove globally random noise from images to within the accuracy of which the human eye can detect. Gibbs oscillations are not random noise as they possess a definite structure. However, we have 
shown through numerical examples that the DTV filter is indeed effective in post-processing pseudospectral approximations. Without the need for separate edge detection methods, the DTV filter sharply resolves discontinuities and accelerates the overall convergence of an oscillatory pseudospectral approximation in a robust, computationally efficient manner. For CPS SSV approximations, which already have been weakly filtered, the DTV filter can be applied with a constant global $\lambda$ without additional spectral filtering. Otherwise, for approximations such as interpolation problems and linear hyperbolic PDEs to which filtering has not been applied, the DTV filter is most successful if the oscillatory solution if first filtered with a weak spectral filter or is applied in a hybrid manner with DTV filter being used in regions of strong oscillations and a spectral filter in smoother regions away from discontinuities.

DTV filtering is a physical space filtering process that can be applied to data on arbitrary grids. If the data is widely scattered, some modifications to the filtering process may be necessary. For the non-uniform but highly structured non-periodic pseudospectral grids, DTV filtering many be applied without regard to the varying distance between data points. In this work we have focused on postprocessing Chebyshev pseudospectral approximations, but the results apply equally as well to other nonperiodic pseudospectral methods, e.g. Legendre pseudospectral methods. Interior jumps in periodic data approximated on a uniform grid by a Fourier pseudospectral method can be post-processed by the DTV filter as well. However, the DTV filter is not effective for jumps at the boundary caused by the data being nonperiodic.

If a truly "black box" post-processing method is to be implemented, the idea of DTV filtering with its built-in edge recognition, or a combination of DTV and spectral filtering, are excellent candidates. At the very least, the DTV filter as a post-processing for pseudospectral methods is a very good choice when other existing methods, such as the Gegenbauer Reconstruction procedure, either fail, are cumbersome to apply, or are too computationally expensive. This is often the case for pseudospectral solutions to conservation laws, such as the fluidized bed equations with source term in one-dimension, and most equations in higher dimensions.

\section{References}

[1] J. P. Boyd. Chebyshev and Fourier Spectral Methods. Dover Publications, Inc, New York, second edition, 2000. 
[2] A. Burgel, T. Grahs, and T. Sonar. From continuous recovery to discrete filtering in numerical approximations of conservation laws. Applied Numerical Mathematics, 42:47-60, 2002.

[3] A. Burgel and T. Sonar. Discrete filtering of numerical solutions to hyperbolic conservation laws. International Journal for Numerical Methods in Fluids, 40:263-271, 2002.

[4] C. Canuto, M. Y. Hussaini, A. Quarteroni, and T. A. Zang. Spectral Methods for Fluid Dynamics. Springer-Verlag, New York, 1988.

[5] T. Chan, S. Osher, and J. Shen. The digital TV filter and nonlinear denoising. IEEE Transactions on Image Processing, 10(2), 2001.

[6] I. Christie, G.H. Ganser, and J.M. Sanz-Serna. Numerical solution of a hyperbolic system of conservation laws with source term arising in a fluidized bed model. Journal of Computational Physics, 93(2):297-311, 1991.

[7] I. Christie and C. Palencia. An exact Riemann solver for a fluidized bed model. IMA Journal of Numerical Analysis, 11:493-508, 1991.

[8] A. Gelb. A hybrid approach to spectral reconstruction of piecewise smooth functions. Journal of Scientific Computing, 15:293-322, 2001.

[9] A. Gelb and E. Tadmor. Detection of edges in spectral data. Applied and Computational Harmonic Analysis, 7:101-135, 1999.

[10] A. Gelb and E. Tadmor. Detection of edges in spectral data II: Nonlinear enhancement. SIAM J. Numer. Anal., 38:1389-1408, 2000.

[11] A. Gelb and E. Tadmor. Enhanced spectral viscosity approximations for conservation laws. Applied Numerical Mathematics, 33:3-21, 2000.

[12] A. Gelb and E. Tadmor. Spectral reconstruction of peicewise smooth functions from their discrete data. Mathematical Modelling and $\mathrm{Nu}$ merical Analysis, 36(2):155-175, 2002.

[13] A. Gelb and J. Tanner. Robust reprojection methods for the resolution of the Gibbs phenomenon. To appear in Applied and Computational Harmonic Analysis, 2005.

[14] D. Gottlieb and S. A. Orszag. Numerical Analysis of Spectral Methods. SIAM, Philadelphia, PA, 1977. 
[15] D. Gottlieb and C. Shu. On the Gibbs phenomenon IV: Recovering exponential accuracy in a subinterval from a Gegenbauer partial sum of a piecewise analytic function. Mathematics of Computation, 64:10811095, 1995.

[16] D. Gottlieb and C. Shu. On the Gibbs phenomenon V: Recovering exponential accuracy from collocation point values of a piecewise analytic function. Numerische Mathematik, 71:511-526, 1995.

[17] D. Gottlieb and C. Shu. On the Gibbs phenomenon III: Recovering exponential accuracy in a subinterval from a partial sum of a piecewise analytic function. SIAM Journal of Numerical Analysis, 33:280-290, 1996.

[18] D. Gottlieb and C. Shu. On the Gibbs phenomenon and its resolution. SIAM Review, 39(4):644-668, 1997.

[19] D. Gottlieb, C.-W. Shu, A. Solomonoff, and H. Vandeven. On the Gibbs phenomenon I: recovering exponential accuracy from the Fourier partial sum of a nonperiodic analytic function. Journal of Computational and Applied Mathematics, 43:81-98, 1992.

[20] D. Gottlieb and E. Tadmor. Recovering pointwise values of discontinuous data within spectral accuracy. In E. M. Murman and S. S. Abarbanel, editors, Progress and Supercomputing in Computational Fluid Dynamics, pages 357-375. Birkhäuser, Boston, 1985.

[21] Z. Jackiewicz. Detierminination of optimal parameters for the chebyshev-gegenbauer reconstruction method. SIAM Journal of Scientific Computing, 25(4), 2003.

[22] R. Kosloff and H. Tal-Ezer. A modified Chebyshev pseudospectral method with an $\mathrm{O}(1 / \mathrm{N})$ time step restriction. Journal of Computational Physics, 104:457-469, 1993.

[23] A. Fatemi L. Rudin, S. Osher. In Proceedings of the 11th annual international conference of the Center for Nonlinear Studies on Experimental mathematics, pages 259-268, 1992.

[24] H. Ma. Chebyshev-Legendre super spectral viscosity method for nonlinear conservation laws. SIAM Journal of Numererical Analysis, 35:893908, 1998. 
[25] S. Osher and J. Shen. Digitized PDE method for data restoration. In G. Anastassiou, editor, Analytic-Computational Methods in Applied Mathematics, chapter 16, pages 751-771. Chapman and Hall/CRC, 2000 .

[26] R. Pasquetti. On inverse methods for the resolution of the Gibbs phenomenon. Journal of Computational and Applied Mathematics, 170:303-315, 2004.

[27] S. A. Sarra. Chebyshev super spectral viscosity method for a fluidized bed model. Journal of Computation Physics, 186(2):630-651, 2003.

[28] B. Shizgal and J. Jung. Towards the resolution of the Gibbs phenomena. Journal of Computational and Applied Mathematics, 161:41-65, 2003.

[29] E. Tadmor. Convergence of spectral methods for nonlinear conservation laws. SIAM Journal of Numerical Analysis, 26:30-44, 1989.

[30] E. Tadmor. Numerical methods for fluid dynamics IV. In Proceedings of the 1992 conference on Numerical Methods for Fluid Dynamics, pages 69-82, 1993.

[31] E. Tadmor and J. Tanner. Adaptive mollifers - high resolution recovery of piecewise smooth data from its spectral information. Foundations of Computational Mathematics, 2:155-189, 2002.

[32] J. Tanner. Optimal filter and mollifier for piecewise smooth spectral data. Submitted to Mathematics of Computation, 2004.

[33] L. N. Trefethen. Spectral Methods in Matlab. SIAM, Philadelphia, 2000.

[34] H. Vandeven. Family of spectral filters for discontinuous problems. SIAM Journal of Scientific Computing, 6:159-192, 1991. 


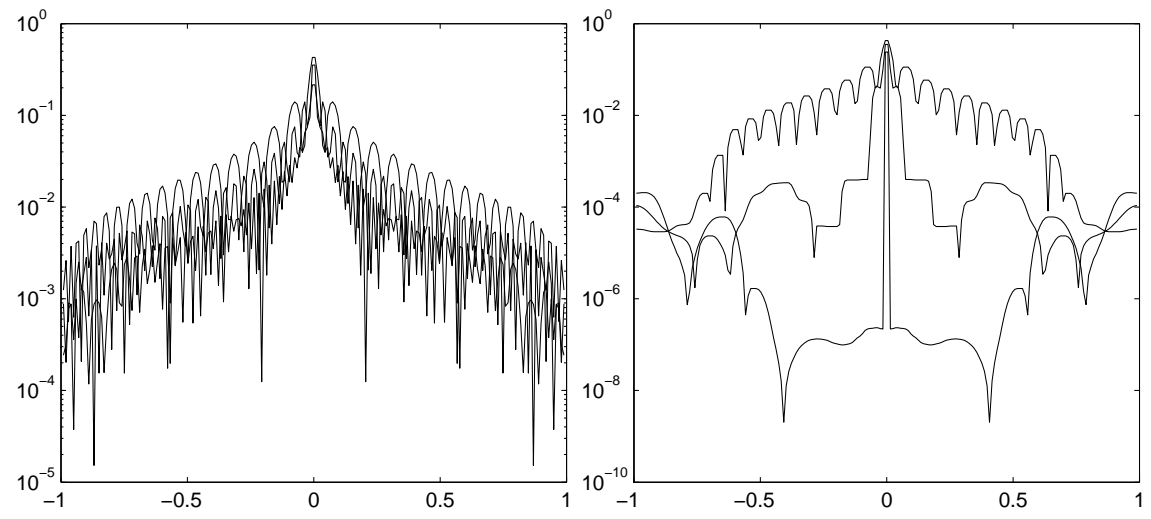

Figure 1: Left: Convergence of the approximation of function (17) for $N=$ $39, N=79$, and $N=159$. Right: Pointwise errors of the DTV postprocessed Chebyshev approximation with $N=39,79,159$ and constant $\lambda=$ 15.
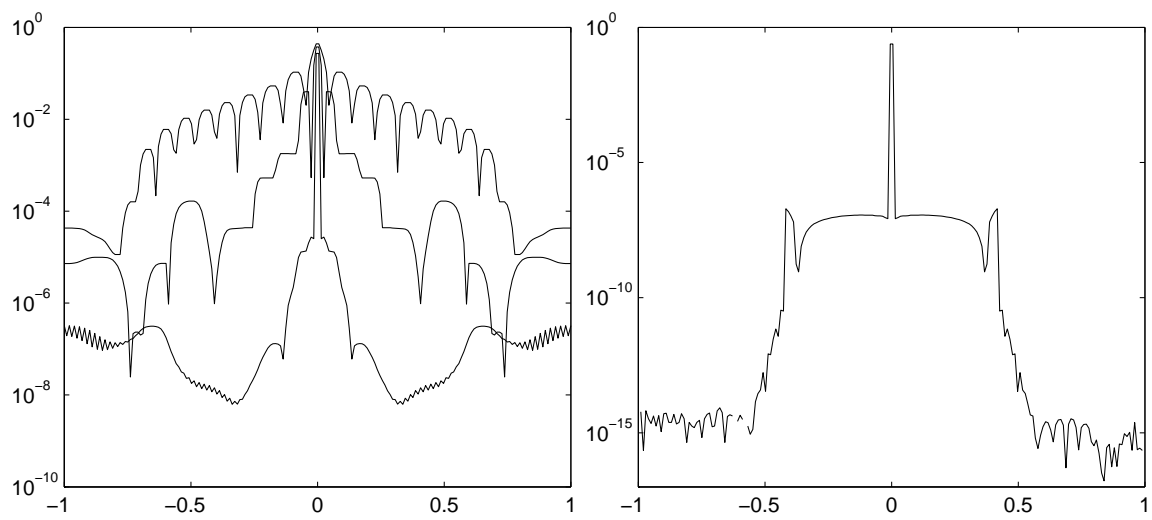

Figure 2: Pointwise errors of the DTV post-processed Chebyshev approximation with $N=39,79,159$. Left: Exponential filter with $p=32$ and then DTV filtering with constant $\lambda=15$. Right: Hybrid DTV-spectral post-processing, $\lambda=15, p=4$, and $t o l_{p p}=10 \mathrm{e}-3$. 

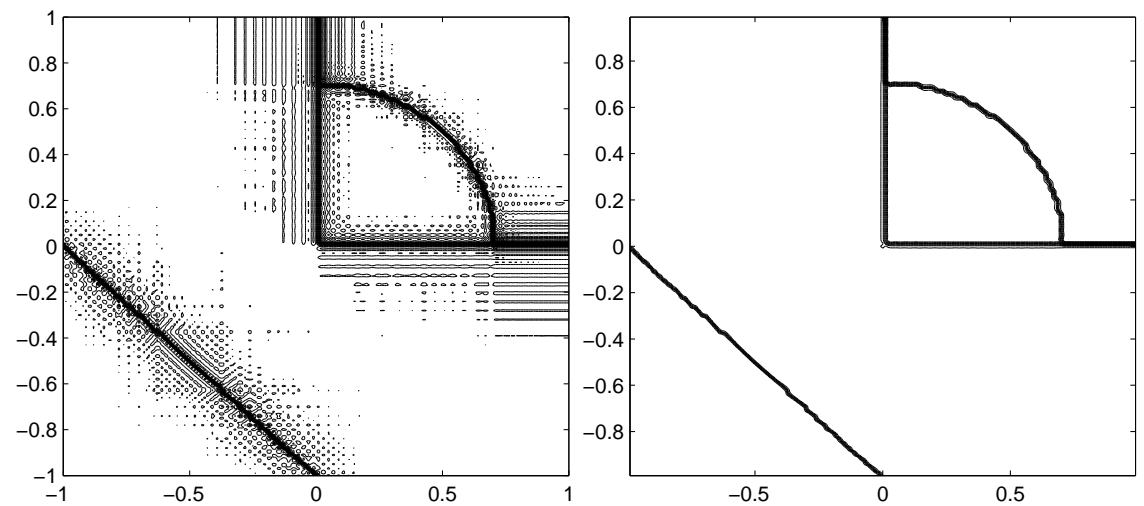

Figure 3: Left: Chebyshev approximation of (18), $N=160$. Right: Hybrid, $\lambda=9$ and 50 iterations, $p=4, t l_{p p}=10 \mathrm{e}-3$.
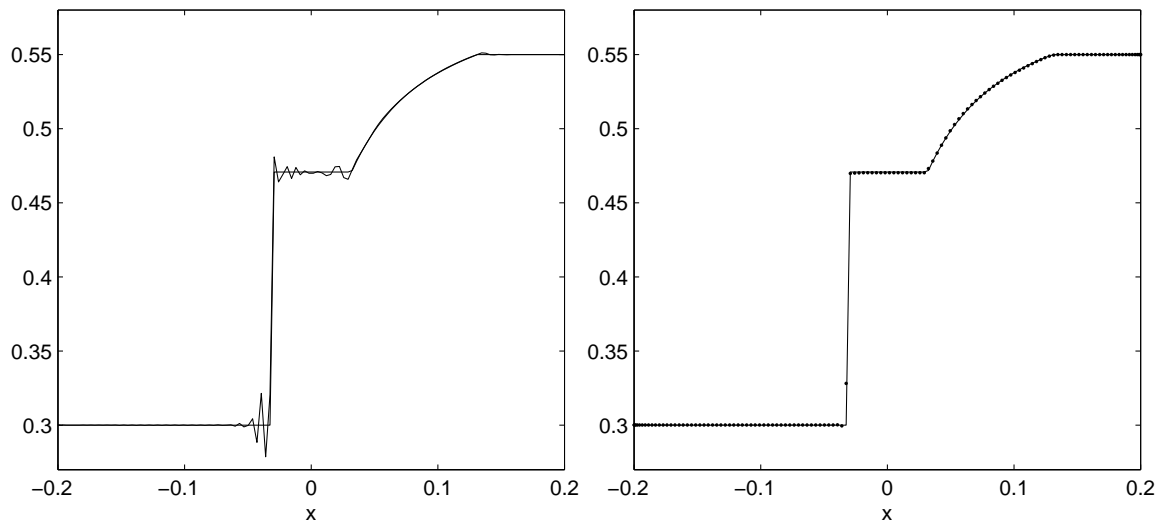

Figure 4: Left: Homogeneous fluidized bed Chebyshev SSV approximation (oscillatory) vs. exact. Right: DTV post-processed (solid dots) fluidized bed concentration solution vs. exact (solid) at time $t=0.5$ using $\lambda=105$ and $t o l=10 e-8$ which leads to 524 iterations taking 0.16 second. 


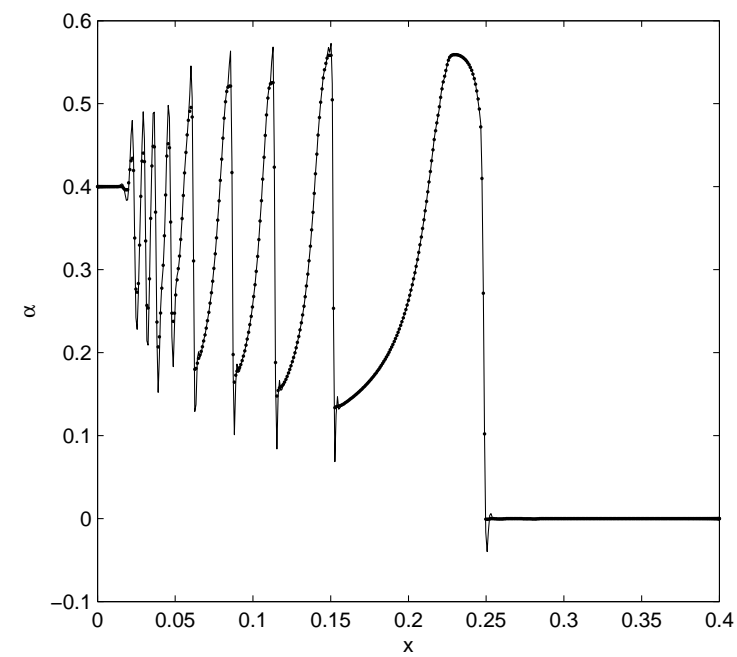

Figure 5: Fluidized bed equations with source term SSV concentration solution with Gibbs oscillations (solid) vs. DTV post-processed (solid dots) at time $t=2.95$ using $\lambda=35$ and 40 iterations taking 0.047 second.

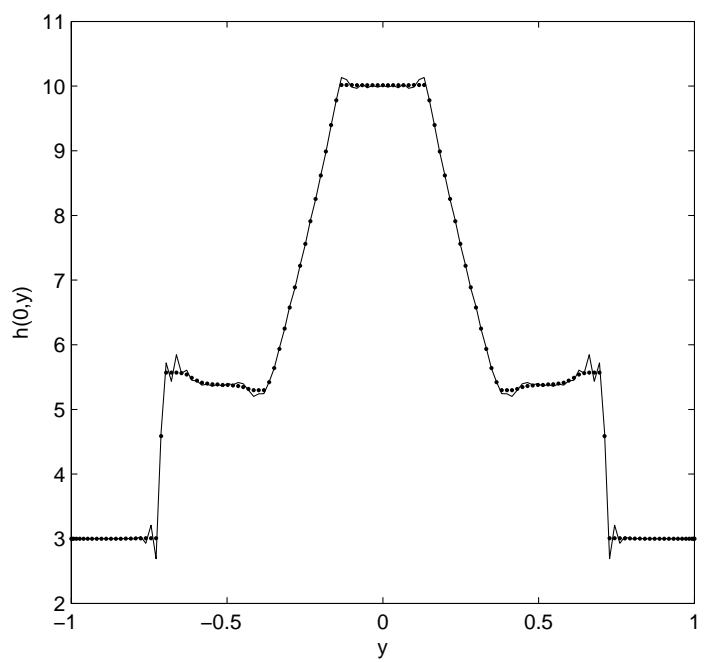

Figure 6: Cross section at $x=0$ of Shallow water height SVV solution (solid) and DTV post-processed (solid dots). DTV parameters of $\lambda=5$ and 40 iterations. 